Nonlinear Processes in Geophysics, 12, 435-439, 2005

SRef-ID: $1607-7946 / \mathrm{npg} / 2005-12-435$

European Geosciences Union

(C) 2005 Author(s). This work is licensed

under a Creative Commons License.

\title{
Effects of the synoptic scale variability on the thermohaline circulation
}

\author{
J. J. Taboada ${ }^{1}$ and M. N. Lorenzo ${ }^{2}$ \\ ${ }^{1}$ Group of Nonlinear Physics, Faculty of Physics, Univ. of Santiago de Compostela, 15782 Santiago de Compostela, Spain \\ ${ }^{2}$ Faculty of Sciences, Campus de Ourense, Univ. of Vigo, 32004 Ourense, Spain
}

Received: 19 November 2004 - Revised: 8 March 2005 - Accepted: 9 March 2005 - Published: 24 March 2005

\begin{abstract}
In this paper the effect of the synoptic scale variability is analyzed using a simple atmosphere-ocean coupled model. This high frequency variability has been taken into account in the model adding white gaussian noise in variables related to zonal and meridional temperature differences. Results show that synoptic scale frequency variability on longitudinal heating contrast between land and sea can produce a collapse of thermohaline circulation when a threshold of noise is overcome. This result is significant because if synoptic scale variability in the next century increases due to the climatic change an increment of the probability of this collapse could be produced.
\end{abstract}

\section{Introduction}

In the last decades it has been established (Rahmstorf, 2000) that termohaline circulation (THC) plays a main role regulating North Atlantic climate. Its formation depends on North Atlantic surface water being sufficiently cold and salty to destabilize water column and produce deep water formation. In this way THC formation is very sensitive to air-sea heat exchange and freshwater input in North Atlantic. On the other hand these two parameters are expected to vary due to climate change produced by greenhouse gases accumulation in the atmosphere. The fate of THC under those new conditions has been the subject of a scientific debate since a work of Broecker (1987). This work warned for the first time about the possibility of a sudden climate change due to a switch in North Atlantic circulation driven by a freshening of surface water that would prevent formation of North Atlantic deep water (NADW). In a posterior work of the same author, Broecker (1997), THC was nominated the Achilles heel of actual climate, highlighting the possibility that minor changes in parameters can cause a sudden change in climate conditions. This hypothesis has been also sustained by the

Correspondence to: J. J. Taboada

(uscfmjth@cesga.es) growing evidence from paleoclimatic archives of past abrupt climate changes (Stocker and Schmittner, 1997).

Many modeling studies have been done in the last decades in order to study the behavior of THC under a changing climate (Stocker and Schmittner, 1997; Rahmstorf, 2000; Stouffer and Manabe, 2003). Results are somewhat different, ranging from the possibility of a total collapse, to a reinforcement of THC, but a common picture appears. This picture shows that ocean-atmosphere system has more than one stable mode of operation. A schematic stability diagram can be found in Rahmstorf (2000), showing the nonlinear characteristic of THC that implies different responses to perturbations. Freshwater input appears as a key control variable for the THC.

The main uncertainties that must be overcome in these studies are, on the one hand to know exactly the actual evaporation-precipitation budget in North Atlantic. We must remind that in nonlinear systems this is a critical question, because depending on how close of the threshold we are, the behavior of the whole system facing the same perturbation will be very different. On the other hand, it is also very important to know the actual intensity of THC (Tziperman, 2000). WOCE project has obtained a value of $15 \pm 2 \mathrm{~Sv}$ that can be used as real value (Ganachaud and Wunsch, 2000).

The increasing rate of freshwater input (Stocker et al., 2001) and the location of this input are also important and unknown variables. Moreover in 3-D models it has been demonstrated that THC behavior depends greatly on vertical mixing parametrization (Knutti et al., 2000).

It is also necessary to know the "hydrological sensibility" (Rahmstorf and Ganopolski, 1999), namely, the rate of change of hydrological cycle in a heating atmosphere. The problem of climate change itself is not yet well established and some quite surprising feedbacks can appear. In this way, Delworth and Dixon (2000) have proposed that in an scenario of global heating, NAO (North Atlantic Oscillation) would be preferably in the positive phase, enhancing North Atlantic winds. These enhanced winds will cause a cooling of surface water that can cancel the effects of freshening. On the other 


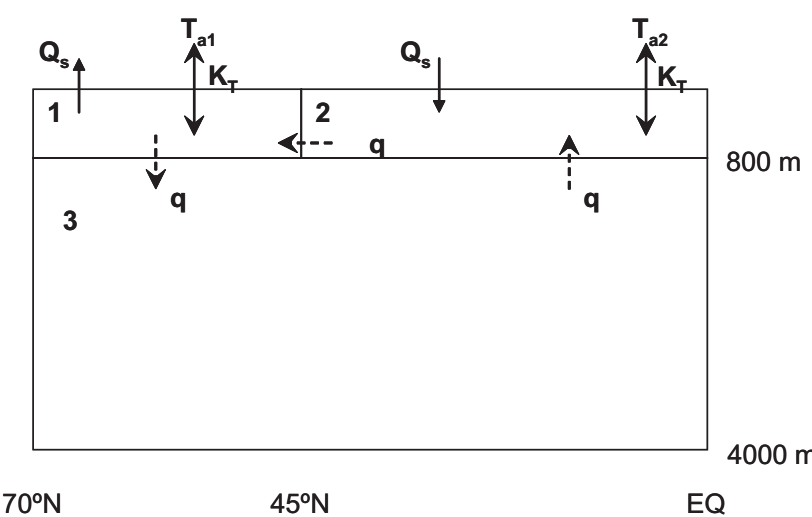

Fig. 1. Geometry of the North Atlantic through a 3-D box model. $q$ represent the thermohaline circulation and the sense of the arrows indicates a positive circulation.

hand, Latif et al. (2000) have proposed a similar mechanism but with ENSO pattern.

In this way, we can see that 3-D fully comprehensive climate models have some uncertainties, in particular in response to freshwater inputs (IPCC, 1999). Moreover, those models do not explicitly simulate synoptic variability, i.e. the main stochastic component of climate system. This unresolved variability can be added as random fluctuations in parameters or variables of the model. The role of these fluctuations could be decisive to regulate the transition between the different operation modes of the THC as occurs in other physical systems (García-Ojalvo and Sancho, 1999; Buceta et al., 2003; Ullner et al., 2003).

In this work we use a low complexity ocean-atmosphere coupled model to investigate the possibility of a collapse of thermohaline circulation taking into account the synoptic atmospheric variability. These kind of models can only give qualitative results. But, the currently available computing capacity reduces the possibility of carry out exhaustive parametric studies of the THC using 3-D models. In this way models of reduced complexity can make valuable contributions to a better understanding of parameter space and they are also useful as an hypothesis builder.

The aim of the paper is to prove the ability of the model to provoke a collapse of the THC under some hypothesis such as a massive sudden freshwater input. Then we will look at the possibility of such a collapse adding noise in order to simulate synoptic variability.

\section{Model}

The atmosphere-ocean model used in this paper has been taken from a previously published work of Roebber (1995). The atmospheric part of the model is represented by a loworder model introduced by Edward Lorenz in 1984 and defined by three equations:

$\frac{d X}{d t}=-Y^{2}-Z^{2}-a X+a F$ $\frac{d Y}{d t}=X Y-b X Z-Y+G$

$\frac{d Z}{d t}=b X Y+X Z-Z$,

where $X, Y$ and $Z$ represent the meridional temperature gradient and the amplitudes of the cosine and sine phases of a chain of superposed large scale eddies, respectively. In Eq. (1), $F$ represents the meridional gradient of diabatic heating and in the Eq. (2) $G$ is the asymmetric thermal forcing, representing the longitudinal heating contrast between land and sea, $a=0.25$ and $b=4$.

The ocean model considered is a three box model (Fig. 1) representing thermohaline circulation in the North Atlantic ocean. In this model, $Q_{s}$ denotes the equivalent salt flux (differential net surface evaporation), $T_{a 1}$ and $T_{a 2}$ represent restoring air temperatures with restoring constant $K_{T}$, and $q$ is the magnitude of the thermohaline circulation. The sense of the circulation indicated denotes $q$ positive. The explicit equations of the model and the ocean model constants are written in the work of Roebber (1995).

The atmospheric model is coupled to the ocean model through $F$ and $G$ that are allowed to vary in a seasonal cycle

$$
\begin{aligned}
& F(t)=F_{0}+F_{1} \cos \omega t+F_{2}\left(T_{2}-T_{1}\right) \\
& G(t)=G_{0}+G_{1} \cos \omega t+G_{2} T_{1},
\end{aligned}
$$

where $\omega$ is the annual frequency and $t=0$ at winter solstice. The values chosen are, $F_{0}=4.65, F_{1}=1, F_{2}=47.9$, $G_{0}=-3.60, G_{1}=1.0$ and $G_{2}=4.0254$. These assumptions insure that $F$ and $G$ remain bounded by qualitatively plausible constraints for collapse flows (Roebber, 1995). On the other hand, the ocean is coupled to the atmosphere through the restoring temperatures $T_{a 1}$ and $T_{a 2}$ and the equivalent salt flux (differential net surface evaporation) $Q_{s}$ with the next expressions:

$$
\begin{aligned}
T_{a 1}(t) & =T_{a 2}-\gamma X(t) \\
Q_{s}(t) & =0.00166+0.00022\left(Y^{2}+Z^{2}\right),
\end{aligned}
$$

where $\gamma=0.06364$ and $T_{a 2}=25^{\circ} \mathrm{C}$ are constants and the parametrization of $Q_{s}(t)$ is due to the assumption that the eddy water vapor transport is directly proportional to the eddy sensible heat flux given by $Y^{2}+Z^{2}$.

The Lorenz model that simulates atmosphere with the chosen parameters has a chaotic behavior. The spectrum of variability of this model is the typical one corresponding to a chaotic system. However, synoptic-scale spectrum is very different from chaotic spectrum (Gulev et al., 2002) and therefore the output of the Lorenz model can not be interpreted as synoptic scale variability in our case. To introduce the synoptic variability inside this model, i.e. the main stochastic component of climate system, we added random fluctuations in an additive way in parameters $F$ and $G$. These fluctuations are given by white Gaussian noise with zero mean and whose correlation function is

$\left\langle\xi_{w}(t) \xi_{w}\left(t^{\prime}\right)\right\rangle=2 A \delta\left(t-t^{\prime}\right)$, 

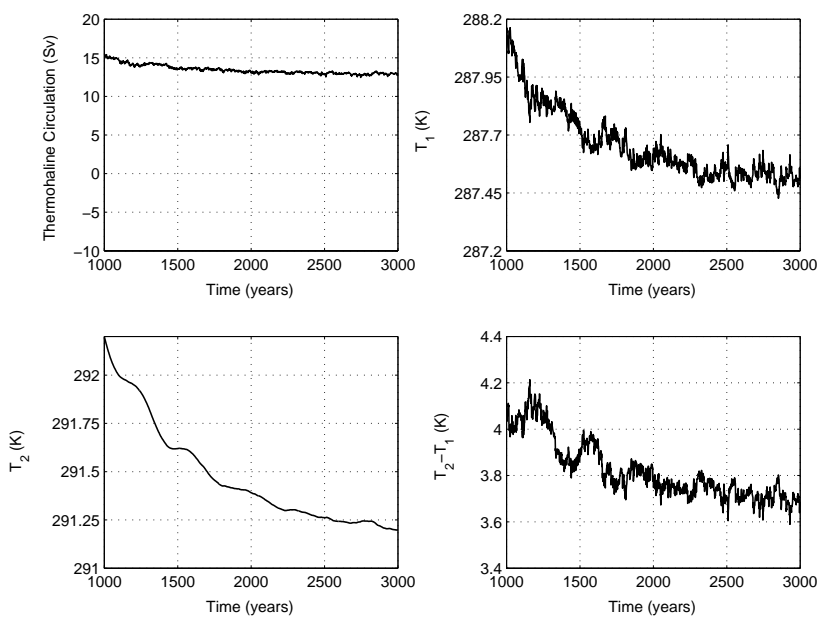

Fig. 2. Present-day steady state of the ocean-atmosphere system with active THC.

where $A$ represents the noise intensity and $2 A$ its variance. We have chosen this kind of noise parametrization because we are looking for the possibility that processes of a frequency higher than the considered in the model could provoke a collapse in thermohaline circulation. Moreover, the effect of atmospheric processes on the ocean has been taken into account in previous work as white noise (Frankignoul and Hasselmann, 1977).

\section{Results}

In a first study of the model we looked for the present-day state of the ocean-atmosphere system. This steady-state has an average temperature of $287.5 \mathrm{~K}$ in north hemisphere latitudes ranging from $70^{\circ} \mathrm{N}$ to $45^{\circ} \mathrm{N}$ and $291 \mathrm{~K}$ in latitudes from $45^{\circ} \mathrm{N}$ to the equator. The strength of the THC is about $15 \mathrm{~Sv}$ (Fig. 2). This value was obtained changing the vertical exchange parameters in the ocean model in order to obtain a value near the measurements from hydrographical data gathered in the WOCE (World Ocean Circulation Experiment) (Ganachaud and Wunsch, 2000). The small variations observed in Fig. 2 are negligible versus a collapse of the THC.

After the obtention of the present-day state, we searched for the possibility of a collapse of the THC under a massive sudden freshwater input. In Fig. 3 we can see how THC collapses when we simulate a massive freshwater input. In this simulation, the equivalent salt flux of box $1, Q_{s 1}$, was forced to take a value greater than in the box $2, Q_{s 2}$, in order to simulate a Younger Dryas-like episode. In this figure we can see how THC collapses one hundred years after this process begins and when the model returns to present-day conditions, $Q_{s 1}=Q_{s 2}=Q_{s}(t)$, THC does not recover in a next future. This result means that the model is able to simulate a collapse of THC under feasible hypothesis like a Younger Dryas episode.
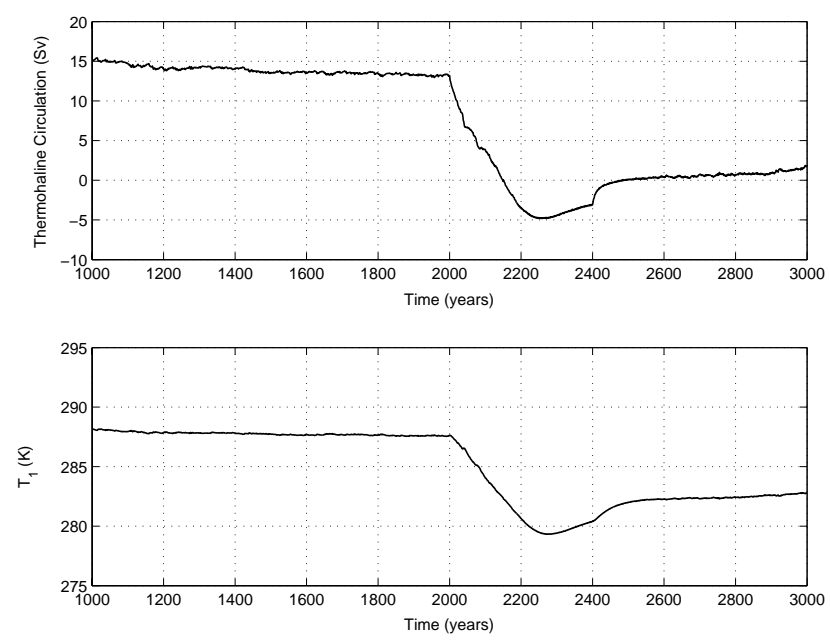

Fig. 3. Effects of a massive freshwater input. $Q_{s}=0.008$ between 2000 and 2400 years, $Q_{s 2}$ is given by equation (7).

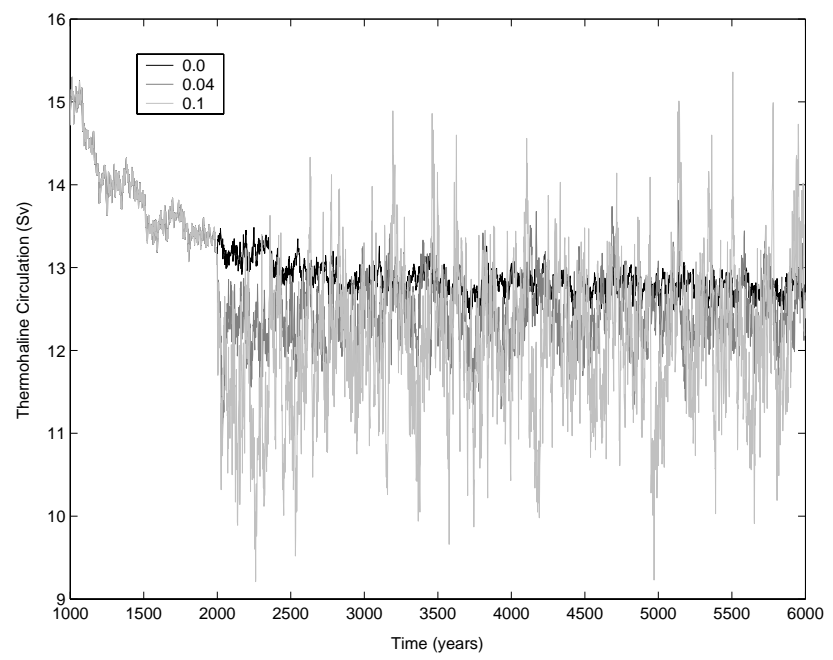

Fig. 4. Effects of a stochastic perturbation on the meridional gradient of diabatic heating $F$ for different values of the intensity of the applied noise $A$.

The main objective of this work is to consider if highfrequency variations in the meridional gradient of diabatic heating or in the longitudinal heating contrast between land and sea could induce significant changes in the ocean circulation. To carry out this study we run the model under present-day conditions (Fig. 2) with $Q_{s 1}=Q_{s 2}=Q_{s}(t)$. To simulate this variation we must remind that when modeling the low-frequency behavior of the climate system, the higherfrequency components can be parameterized as stochastic variables. In this way, we introduced stochastic perturbations of white gaussian noise in the parameters $F$ and $G$ of the model in an additive way and we analyzed their effects. In Fig. 4 we show the behavior of thermohaline circulation when the noise is added in $F$. In this case THC experiments a greater variability but it oscillates around $15 \mathrm{~Sv}$ and we don't achieve a collapse of the THC. This result seems 


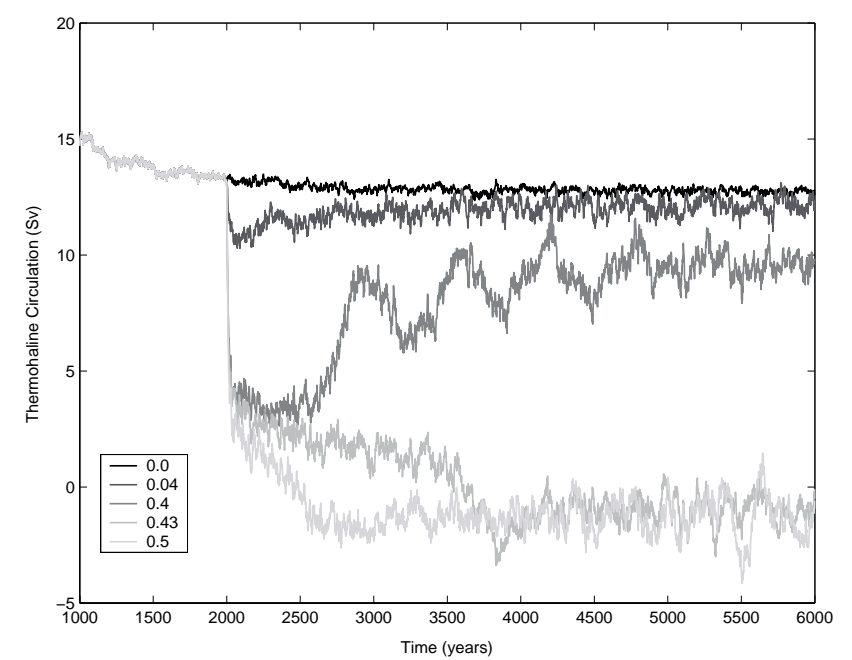

Fig. 5. Effects of a stochastic perturbation on the zonal gradient in diabatic heating $G$ for different values of the intensity of the applied noise $A$.

understandable. $F$ enters in Lorenz equation modifying meridional temperature gradient $X$. In this way we can see in Eq. (6) that this "noisy" $X$ introduces noise in the difference between temperature $T_{a 1}$ and $T_{a 2}$. We must take into account that this difference is by far greater than the noise introduced. Increments or decrements between northern and southern latitudes in North Hemisphere could strengthen or weaken the THC but a collapse would be very difficult because the basic differences between north and south are maintained. However, if we introduce stochastic forcing in $G$ we can detect a collapse of THC, when the stochastic component is sufficiently great. In Fig. 5 we can see that with the increment of the intensity of the forcing on $G$ the THC is destabilized and after passing through an oscillatory transient state, with a frequency which corresponds to the diffusive time-scale of the ocean circulation years (Roebber, 1995), it collapses completely. This collapse is related with the salinity in the ocean because the parameter $G$ is introduced in salt flux $Q_{s}$ and in this case noise can be compared in magnitude with salt flux, meaning that it will be more significant. $G$ represents the difference between the temperature in the land and the temperature in the ocean. An increment in the temperature of the land can provoke a net freshwater flux into the northern part of the North Atlantic with the consequent loss of salinity and the collapse of THC. In the real climate that means that it is easier to collapse thermohaline circulation, changing the budget evaporation-precipitation, than changing differences in temperatures. In other words, although the global warming by itself will not be able to collapse this circulation, the indirect effects (sudden freshwater inputs, more synoptic variability...) could help this collapse to be reached. It is important to remember that now noise is comparable to the signal. This means that if variability in the synoptic scale is comparable to variability in the climatic scale, THC could be eventually driven to a collapse.

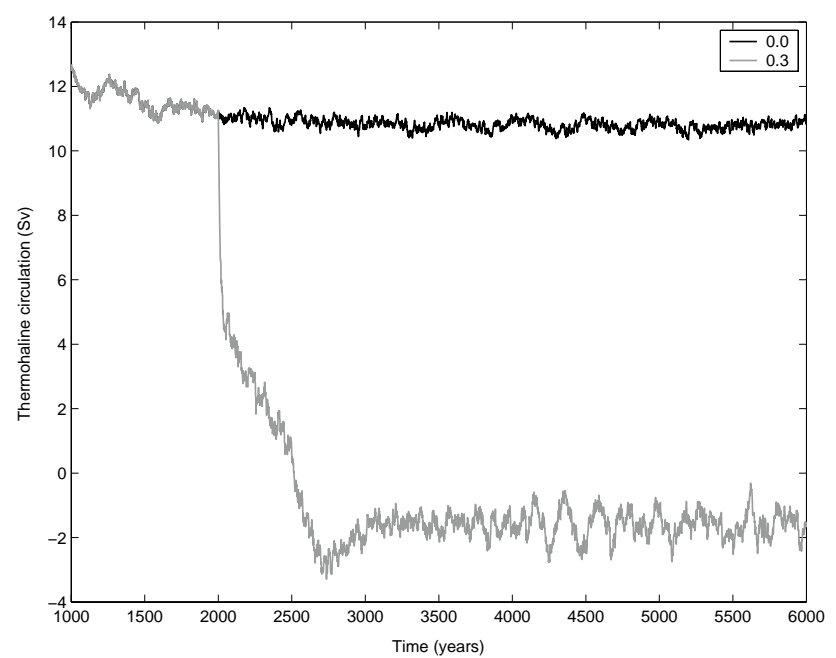

Fig. 6. Effects of a stochastic perturbation on the zonal gradient in diabatic heating $G$ after a weakening of the THC due to a increasing of the $\mathrm{CO}_{2}$ concentration for two values of the intensity of the applied noise $A$.

Finally, owing to the fact that THC is very sensitive to parameters like atmospheric greenhouse gases concentration we have also analyzed the consequences of an increment of these gases. It has been established (IPCC, 1999) that temperature differences between polar and equatorial latitudes will decrease in a global warming context. In this way, we simulate the fate of THC under global warming, decreasing the value of $\gamma$ that represents in the model those differences. In Fig. 6 we can see that these decreasing differences in temperature provoke a weakening in THC that eventually could achieve a total collapse if a threshold value is exceeded. But, even if this threshold is not exceeded in the next century, the weakening of THC implies that high-frequency synoptic variations could provoke a collapse in THC easier than in actual conditions, as shown also in Fig. 6, where the necessary intensity noise to obtain a collapse of the THC is lower than in the Fig. 5.

\section{Conclusions}

Freshwater input seems to be a key parameter controlling THC behavior (Rahmstorf, 2000). The possibility of a massive sudden freshwater input arising from disintegrating land ice sheets and mountain glaciers, must be taken into account because those massive inputs have been documented over some periods in the geological history (Clark et al., 2001). Those bursts of freshwater will stabilize the upper water column and will affect deep water formation, reducing the ability of its water to sink.

In this work, with a simple model, we can see that with an reasonable hypothesis, that increases freshwater input in North Atlantic in the same rate as did in Younger Dryas, THC undergoes a collapse that decrease average temperatures in 
North Atlantic by $5^{\circ} \mathrm{C}$. This test has been done to prove the ability of the model to simulate an eventual collapse of THC.

The next step of the study was to consider synoptic variability inherent in all climate studies. This variability was simulated adding white noise to parameters of the model that take into account meridional and zonal temperature variation. It is important to note that lack of predictability near thresholds implies that abrupt climate changes will always have more uncertainty than gradual climate change and highfrequency variability represented by white noise can take a main role in this kind of systems as has been demonstrated in other works (Ganopolski and Rahmstorf, 2002). In our case noise added to the parameter that represents meridional temperature variation does not have relevant effects in THC circulation. But noise added in the parameter that represents zonal temperature variation can cause a collapse of THC circulation. Although in actual conditions high-frequency variability is not able to collapse THC if this circulation is progressively weakening by global warming, the intensity of noise necessary to provoke the collapse diminishes and this synoptic variability could help THC to collapse. On the other hand, climate change will have as a consequence an increase in extreme events, increasing in this way the synoptic variability.

As a general conclusion we could state that we have used a simple model that gives only qualitative results, but can be taken into account as hypothesis builder. The hypothesis of this work is that the probable increase of synoptic variability in the next century can contribute to an eventual collapse of the THC.

Acknowledgements. Financial support from the Department of Environment of the Galician Government (Xunta de Galicia) is gratefully acknowledged.

Edited by: A. Provenzale

Reviewed by: two referees

\section{References}

Broecker, W. S.: Unpleasant surprises in the greenhouse, Nature, 328, 123-127, 1987

Broecker, W. S.: Thermohaline Circulation, the Achilles' Heel of Our Climate System: Will Man-Made $\mathrm{CO}_{2}$ Upset the Current Balance?, Science, 278, 1582-1588, 1997.

Buceta, J., Ibañes, M., Sancho, J. M., and Lindenberg, K.: Noisedriven mechanism for pattern formation, Phys. Rev. E, 67, 021113 (1-8), 2003.

Clark, P., Marshall, S., Clarke, G., Hostetler, S., Licciardi, J., and Teller, J.: Freshwater Forcing of Abrupt Climate Change During the Last Glaciation, Science, 293, 283-287, 2001.
Delworth, T. L. and Dixon, K. W.: Implications of the recent trend in the Arctic/North Atlantic Oscillation for the North Atlantic thermohaline circulation, J. of Climate, 13, 3721-3727, 2000.

Frankignoul, C. and Hasselmann K.: Stochastic Climate Models, II. Application to SST Anomalies and Thermocline Variability, Tellus, 29, 289-305, 1977.

Ganachaud, A. and Wunsch, C.: Improved estimates of global ocean circulation, heat transport and mixing from hydrographic data, Nature, 408, 453-457, 2000.

Ganopolski, A. and Rahmstorf, S.: Abrupt Glacial Climate Changes due to Stochastic Resonance, Phys. Rev. Lett., 88, 038 501, 2002.

García-Ojalvo, J. and Sancho, J. M.: Noise in spatially extended systems, Institute for Nonlinear Science, Springer-Verlag, New York, 1999.

Gulev, S. K., Jung. T., and Ruprecht, E.: Climatology and Interannual Variability in the Intensity of Synoptic-Scale Processes in the North Atlantic from the NCEP-NCAR Reanalysis Data, J. of Climate, 15, 8, 809-828, 2002.

IPCC: Climate change 2001: the scientific basis, in: Contribution of Working Group I to the Third Assessment Report of the Intergovernmental Panel on Climate Change, edited by: Houghton, J. T. et al., Cambridge University Press, Cambridge, UK, 881, 2001.

Knutti, R., Stocker, T. F., and Wright, D. G.: The effects of subgridscale parameterizations in a zonally averaged ocean model, J. Phys. Oceanogr., 30, 2738-2752, 2000.

Latif, M., Roeckner, E., Mikolajewicz, U., and Voss, R.: Tropical Stabilization of the Thermohaline Circulation in a Greenhouse Warming Simulation, J. of Climate, 13, 1809-1813, 2000.

Rahmstorf, S.: The thermohaline ocean circulation - a system with dangerous thresholds?, Clim. Change 46, 247-256, 2000.

Rahmstorf, S. and Ganopolski, A.: Long-term global warming scenarios computed with an efficient coupled climate model, Clim. Change, 43, 353-367, 1999.

Roebber, P. J.: Climate variability in a low-order coupled atmosphere-ocean model, Tellus, 47A, 473-494, 1995.

Stocker, T. F., Knutti, R., and Plattner, G. K.: The oceans and rapid climate change: past, present, and future, Geophysical Monograph, 126, AGU, Washington D.C., USA, 2001.

Stocker T. F. and Schmittner, A.: Influence of $\mathrm{CO}_{2}$ emission rates on the stability of the thermohaline circulation, Nature, 388 , 862-865, 1997.

Stouffer, R. J. and Manabe, S.: Equilibrium response of thermohaline circulation to large changes in atmospheric $\mathrm{CO}_{2}$ concentration, Clim. Dyn., 20 , 759-773, 2003.

Tziperman, E.: Proximity of the present-day thermohaline circulation to an instability threshold, J. Phys. Oceanogr., 30, 90-104, 2000 .

Ullner, E., Zaikin, A., García-Ojalvo, J., and Kurths, J.: NoiseInduced Excitability in Oscillatory Media, Phys. Rev. Lett., 91, 180601 (1-4), 2003. 